\section{Revue de Sémantique et Pragmatique}

35-36 | 2015

Interface sémantique/morphologie

\title{
Cas de synonymie entre verbes préfixés en dé et simplex : proposition d'analyse sémantique synchronique
}

Cases of synonymy between a verb and its prefixed counterpart: A synchronic semantic analysis

Pierre Jalenques

\section{(2) OpenEdition \\ Journals}

\section{Édition électronique}

URL : http://journals.openedition.org/rsp/1529

DOI : $10.4000 /$ rsp. 1529

ISSN : 2610-4377

\section{Éditeur}

Presses universitaires d'Orléans

\section{Édition imprimée}

Date de publication : 1 mars 2015

Pagination : 81-104

ISSN : 1285-4093

Référence électronique

Pierre Jalenques, «Cas de synonymie entre verbes préfixés en dé et simplex : proposition d'analyse sémantique synchronique », Revue de Sémantique et Pragmatique [En ligne], 35-36 | 2015, mis en ligne le 01 mars 2016, consulté le 09 mai 2020. URL : http://journals.openedition.org/rsp/1529 ; DOI : https://doi.org/10.4000/rsp. 1529 


\section{CAS DE SYNONYMIE ENTRE VERBES PRÉFIXÉS EN DÉ ET SIMPLEX : PROPOSITION D'ANALYSE SÉMANTIQUE SYNCHRONIQUE}

Pierre Jalenques, Laboratoire Dysola (EA 4701), Université de Rouen.

\section{INTRODUCTION}

Nous nous intéressons ici au problème de la compositionnalité du sens dans le domaine des préfixes verbaux en français. Nous examinerons un type de faits généralement peu ou pas traités dans les études de morphologie synchronique sur les préfixes dérivationnels, à savoir les cas où la contribution sémantique du préfixe au sens du mot apparaît intuitivement opaque. Nous nous intéressons au préfixe dé(s)- (noté dorénavant DÉ), lorsqu'il s'applique à une base verbale ${ }^{1}$. Nous aborderons des verbes comme découper ou déverser qui peuvent être perçus comme synonymes des simplex couper, verser, comme dans les deux exemples suivants :

(1) a. Éplucher les 2 pommes golden et les couper en petits morceaux.

b. Éplucher les 2 pommes golden et les découper en petits morceaux.

(2) a. au japon c'est 11500 tonnes d'eau faiblement radioactives qui avaient été versées dans l'océan

b. au japon c'est 11500 tonnes d'eau faiblement radioactives qui avaient été déversées dans l'océan

Pour un panorama sur les rapports catégoriels associés au préfixe DÉ-, voir Amiot 


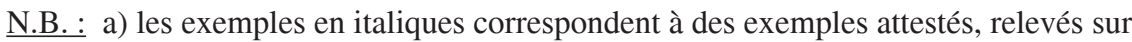
internet ;

b) nous entendrons par acceptions les différentes significations intuitivement attribuées par les locuteurs à une unité perçue comme polysémique ;

c) dans la suite de l'article nous noterons « $\mathrm{V}_{\mathrm{DÉ}}$ » tout verbe dont le signifiant comporte la forme préfixale DÉ-, sans a priori sur le statut morphémique ou non de cette forme préfixale dans le verbe considéré ;

d) $[\mathrm{DHLF}]=$ Dictionnaire Historique de la Langue Française, (1995), éd. Le Robert.

En raison même de cette synonymie avec le simplex, le sens du préfixe est non seulement opaque (découper ne signifie pas le contraire de couper, par différence avec par exemple découdre), mais il apparaît surtout sans contenu sémantique. En raison de ce vide sémantique intuitivement perçu, on considère généralement que découper n'est pas constitué en synchronie du préfixe DÉ (cf. Gerhard (2000), Apothéloz (2007). A ce titre, de tels verbes sont habituellement exclus des études consacrées au préfixe DÉ actuel.

Dans un premier temps, nous montrerons que les verbes comme découper ne peuvent pas être écartés d'une étude synchronique du préfixe DÉ en français actuel. Dans un deuxième temps, nous proposerons une analyse sémantique permettant à la fois de rendre compte de la synonymie perçue entre découper et couper et de montrer que le DÉ actuel est bien présent sémantiquement dans ces verbes, malgré son opacité.

\section{LES DONNÉES DIACHRONIQUES ET SYNCHRONIQUES ACTUELLES CONDUISENT À INTÉGRER LES $V_{\text {DÉ }}$ SYNONYMES DANS L'OBJET D'ÉTUDE}

Avant de poursuivre la description des données évoquées ci-dessus, arrêtons-nous sur la description sémantique du préfixe DÉ en français actuel dans ses emplois sémantiquement transparents, c'est-à-dire les emplois où la compositionnalité du sens est directement accessible à l'intuition des locuteurs. Dans les cas où la base du $\mathrm{V}_{\mathrm{DÉ}}$ est elle-même un verbe, on peut, à la suite de Gerhard-Krait (2000 : 161), identifier trois acceptions principales du préfixe DÉ, qui s'avère donc être polysémique :

1) inversion du résultat exprimé par la base verbale (en lien avec ses compléments éventuels) :

- dénouer sa cravate $=$ agir de telle sorte qu'on annule le résultat du procès « nouer la cravate », c'est-à-dire annuler le résultat « la cravate est nouée ».

2) l'inverse du procès non résultatif exprimé par la base :

- décroître $=1$ 'inverse de crôitre ;

3) la négation du procès non résultatif exprimé par la base :

- déplaire $=$ ne pas plaire. 


\subsection{UN PROBLĖME DE DÉLIMITATION DE L'OBJET D'ÉTUDE}

Lorsque l'on veut établir la liste des $\mathrm{V}_{D E}$ retenus pour l'étude sémantique de DÉ, on constate tout d'abord que ce n'est pas parce qu'un verbe du français présente la forme dé(s) à son initiale qu'il a un quelconque rapport avec le préfixe DÉ ; ainsi des verbes décorer, décupler, sans rapport étymologique avec ce préfixe. Dès lors, on ne peut pas établir la liste des $\mathrm{V}_{\mathrm{DÉ}}$ à retenir à partir de considérations seulement morphophonologiques.

Mais, on le sait, le seul recours au critère étymologique ne suffit pas non plus, puisque nous risquons de « regroupe[r], sur des critères étymologiques, des mots qui n'entretiennent plus actuellement de relation sémantique » (D. Corbin, 1989a : 53). Or, un $V_{D E ́}$ peut a priori ne plus entretenir de relation sémantique avec le préfixe DÉ actuel pour au moins deux raisons bien différentes :

a) la forme préfixale dans ce $V_{D E ́}$ est porteuse d'une valeur sémantique qui ne correspond pas / plus à celle du préfixe actuel. Gerhard-Krait (2000 : 159) mentionne la valeur « agir en tous sens », issue de l'étymon latin du préfixe DÉ, que l'on aurait dans le verbe se démener ;

b) la forme préfixale n'est porteuse d'aucune valeur sémantique identifiable (cf. les verbes découper, déverser vus ci-avant).

Nous n'aborderons ici que le cas b). Dans découper, le problème n'est pas que la forme préfixale serait porteuse d'une signification qui ne correspond plus à celle du DÉ actuel ; le problème, c'est qu'intuitivement il est difficile d'attribuer une quelconque valeur sémantique à cette forme préfixale. Dans cette situation, nous n'avons pas affaire à un problème d'irrégularité sémantique, nous avons affaire à une absence de sens, à première vue en tout cas.

Ainsi, du point de vue de la compositionnalité du sens, parmi les $\mathrm{V}_{\mathrm{DÉ}}$ ayant un lien étymologique avec DÉ, nous avons trois grands types de verbes :

- classe 1: des $\mathrm{V}_{\mathrm{DÉ}}$ dont la compositionnalité du sens est totalement transparente (dénouer, découdre, désapprendre);

- classe 2: des $\mathrm{V}_{\mathrm{DÉ}}$ dont le sens est compositionnel par rapport à la base, mais opaque pour la forme préfixale (découper, déverser, délaisser, dévider). Par exemple, le verbe découper a un rapport sémantique évident avec le verbe couper, mais le sens de la forme préfixale y est opaque.

- classe 3: des $\mathrm{V}_{\mathrm{DÉ}}$ dont la compositionnalité du sens est totalement opaque, autant par rapport à la forme radicale que par rapport au segment préfixal (démanger, déterminer, définir). Par exemple, on ne perçoit pas intuitivement le verbe définir comme composé de dé- et finir. 
Notons que les frontières entre ces trois classes de $V_{\text {DÉ }}$ sont loin d'être nettes et que la présentation donnée ici est simplifiée. En particulier, entre les classes 2 et 3 , on a plutôt une variation progressive de l'opacité du sens, au grès des verbes que l'on envisage. Par exemple, le rapport sémantique entre dénoter et noter est moins clair qu'entre découper et couper mais plus clair qu'entre définir et finir; quand on dénote, on se fait en quelque sorte «noter» au sens de « remarquer, repérer », comme dans notez bien que ...

L'existence de ces trois classes n'est pas spécifique au préfixe DÉ. Jalenques (2001) signale que l'on relève les trois mêmes classes pour les verbes ayant un lien étymologique avec le préfixe RE :

- classe 1 : refaire, retendre, revoir, réécouter

- classe 2 : relier, regrouper, rentrer

- classe 3 : remarquer, remuer, redouter, regarder

Les verbes des classes 2 et 3 ont en commun le fait que, intuitivement, il est difficile d'y attribuer une signification à la forme préfixale.

Dans cet article, les verbes auxquels nous nous intéressons, à savoir ceux qui sont en relation de synonymie avec leur simplex, correspondent à une sousclasse des $\mathrm{V}_{\mathrm{DÉ}}$ de la classe $2^{2}$. Nous avons relevé $22 \mathrm{~V}_{\text {DÉ }}$ pour cette sous-classe ${ }^{3}$ :

- $\mathrm{V}_{\mathrm{DÉ}}$ courants : décalquer, découper, dédoubler, délaisser, délivrer, démultiplier, dénommer, dépasser, dépeindre, déposer, détourner, déverser;

- $\mathrm{V}_{\mathrm{DÉ}}$ peu courants : décarrer (se), déchasser, dégoutter, dégrouiller (se), démaigrir, dépriver, déraser, dessécher, détremper, détripler

On le sait, la synonymie absolue n'existe guère, et il s'agit toujours de voisinage sémantique, c'est-à-dire des mots perçus comme sémantiquement proches, à quelques nuances près (cf. Auroux, 1984). Donc, nous désignons par «synonymes de leur simplex » des $\mathrm{V}_{\mathrm{DÉ}}$ sémantiquement proches de leur simplex, à quelques nuances près. On pourrait ainsi contester la présence de tel ou tel $V_{D E ́}$ dans la liste, la notion de «nuance » étant assez subjective et difficilement quantifiable. Nous n'approfondirons pas ce point ; nous verrons plus loin que l'essentiel n'est pas là. Voici néanmoins quelques exemples

${ }^{2}$ Nous avons relevé en tout 34 verbes pour cette classe. Les 12 autres verbes sont les suivants : débouger (« quitter un endroit»), débouter, déchoir, dégommer (cf. gommer un mauvais souvenir), délaver, démontrer, départager, déperdre, dépérir, dénier, détenir, dévider. Ces verbes ne sont pas synonymes de leur simplex, mais une parenté sémantique est néanmoins claire avec chacun d'eux, comme par exemple entre dénier (à qqn un droit) et le sens de nier, ou bien entre démontrer et montrer. Quantitativement, la sous-classe des $\mathrm{V}_{\mathrm{DE}}$ synonymes de leur simplex constitue le groupe principal de cette classe 2 .

${ }^{3}$ Nous avons extrait ces $V_{D E ́}$ à partir des listes des $V_{D E ́}$ contenus dans le Grand Robert (version électronique) et dans le TLFi. 
supplémentaires concernant des $\mathrm{V}_{D E ́}$ dont la synonymie avec le simplex est intuitivement moins évidente :

(3) 3a. Lorsque vous aurez passé la sortie «Montplaisir», suivez la direction Roanne / Clermont-Ferrand.

b. Lorsque vous aurez dépassé la sortie «Montplaisir», suivez la direction Roanne / Clermont-Ferrand.

[Blog d'élèves présentant un atelier mosaïque dans leur collège]

(4) a. Premièrement, on a calqué un dessin sur la plaque en bois puis on a collé les tesselles sur la plaque.

b. Premièrement, on a décalqué un dessin sur la plaque en bois puis on a collé les tesselles sur la plaque.

(5) a. Il tourna le regard pour éviter de croiser celui de Marie

b. Il détourna le regard pour éviter de croiser celui de Marie

(6) a. 20 sept. 2013 - Mais François Hollande a aussi livré un «message» à la communauté internationale, dans une allusion évidente à la Syrie.

b. 20 sept. 2013 - Mais François Hollande a aussi délivré un «message» à la communauté internationale, dans une allusion évidente à la Syrie.

L'étude de cette sous-classe de $\mathrm{V}_{\mathrm{DE}}$ est intéressante car, d'une part, elle constitue le sous-ensemble principal de la classe 2, d'autre part, la plupart des verbes de la classe 3 ont eux-mêmes été synonymes de leur simplex dans un ancien état de langue. Ainsi, en latin, l'étymon de définir était synonyme de celui de finir, tous les deux pouvant signifier « délimiter, borner, déterminer ». De même, pour le préfixe RE, en ancien français, le verbe regarder était synonyme de garder, qui lui-même à cette époque pouvait signifier « regarder » (cf. le sens du verbe italien actuel gardare de même origine).

En d'autres termes, il y a un lien historique régulier entre la situation des verbes de la classe 2 synonymes de leur simplex et la situation de beaucoup de verbes de la classe 3 totalement opaques. Ainsi, ces $V_{D E ́}$ synonymes de leur simplex constituent à la fois un phénomène central parmi les $V_{D E ́}$ sémantiquement opaques par rapport au préfixe et un lieu d'observation privilégié pour comprendre l'émergence de l'opacité dans la compositionnalité du sens des $V_{\text {DÉ }}$ par rapport au préfixe.

Dans les études de morphologie synchronique, les $\mathrm{V}_{\mathrm{DÉ}}$ des classes 2 et 3 sont généralement écartés, en raison même de la difficulté, voire impossibilité, à attribuer intuitivement une signification à la forme préfixale (cf. Muller (1990), Gerhard-Krait (2000), Apothéloz (2007).

En effet, intuitivement, que veut dire DÉ dans découper ou délaisser? Cependant, la mise à l'écart de tels $\mathrm{V}_{\mathrm{DE}}$ repose sur des hypothèses, généralement non discutées, qu'il s'agit maintenant d'expliciter. 


\subsection{LES DONNÉES HISTORIQUES NE PERMETTENT PAS D'ÉCARTER CES VERBES}

Revenons tout d'abord à la citation de D. Corbin (1989a), à propos $\mathrm{du}$ risque de "regroupe[r], sur des critères étymologiques, des mots qui n'entretiennent plus actuellement de relation sémantique ». Le terme important dans cette citation est «ne ... plus », qui renvoie à l'idée d'une perte de sens au cours du temps. On retrouve cette idée à propos du préfixe DÉ dans (Muller, 1990 : 172) : «il se peut que certains composés aient évolué, perdant au fil des siècles et la transparence de leur composition, et l'idée négative que signifiait le préfixe [DÉ] ». On la retrouve encore dans Apothéloz et Boyé (2004) à propos du préfixe com- dans des verbes comme combattre ou concourir : «Dans la synchronie actuelle, le segment com- de COMBATTRE ne renvoie pas à une fonctionnalité morphologique consistante et clairement identifiable dans le lexique. »

L'idée sous-jacente est donc la suivante : au départ, un mot morphologiquement construit est toujours transparent du point de vue de la compositionnalité du sens ; au fil du temps, cette compositionnalité du sens peut s'atténuer, voire, disparaître, le mot perdant alors son statut de mot construit en synchronie actuelle. En d'autres termes, l'opacité sémantique découle par hypothèse d'un processus diachronique. C'est cette hypothèse qui justifie implicitement la mise à l'écart de verbes comme découper en français actuel : au départ le sens de ce verbe aurait été transparent du point de vue de la présence du préfixe, maintenant, elle est opaque.

Que des relations sémantiques deviennent opaques et perdent toute réalité linguistique au fil du temps, cela est indéniable. Mais toute la question est de savoir si l'ensemble des cas d'opacité sémantique en synchronie, notamment dans le domaine des préfixes verbaux, peuvent et doivent être expliqués par la diachronie. En effet, cette hypothèse diachronique implicite repose davantage sur des a priori sur l'histoire de ces verbes préfixés que sur des faits avérés.

Concernant les $V_{D E ́}$ qui nous intéressent ici, synonymes de leur simplex, les données historiques ne confirment pas le scénario diachronique implicite qui permettrait de les écarter, comme nous allons le voir maintenant.

En effet, sur les $22 \mathrm{~V}_{\text {DÉ }}$ recensés ci-avant comme étant synonymes de leur simplex à l'heure actuelle, 17 d'entre eux étaient déjà synonymes de leur simplex dès les premières attestations recensées dans les dictionnaires historiques. Ainsi, le DHLF indique que le verbe délivrer est issu du latin delīběrare qui signifiait « délivrer », construit sur la base līběrare qui signifiait elle-même « délivrer ; le verbe découper a pour premier emploi la valeur « couper en morceaux » $\left(12^{\mathrm{e}} \mathrm{s}\right.$.) ; le verbe délaisser, $12^{\mathrm{e}} \mathrm{s}$., a d'abord signifié « renvoyer, laisser partir »; le verbe dégoutter signifie dès ses premiers emplois, début $12^{\mathrm{e}} \mathrm{s}$., " couler goutte à goutte »; le verbe déchasser signifiait au départ « chasser, expulser »; 
le verbe déverser, apparu au $18^{\mathrm{e}} \mathrm{s}$., signifie dès ses premières attestations « faire couler (un liquide) d'un lieu dans un autre ».

Concernant les cinq autres verbes, trois ne sont certes pas synonymes de leur simplex au départ, mais leur signification est néanmoins opaque dès le départ du point de vue du préfixe : dépasser a d'abord signifié «aller plus loin que », au $12^{\mathrm{e}} \mathrm{s}$.; deux siècles plus tard, il prend d'ailleurs un sens compositionnel transparent avec le sens de « reculer » (le contraire de passer, au sens d' «avancer»); au $11^{\mathrm{e}} \mathrm{s}$., le verbe détourner signifie «écarter qqn de son chemin, de qqch », et non quelque chose comme « le contraire de tourner / aller tout droit / tourner en sens inverse ». Deux verbes ont une origine incertaine du point de vue de la compositionnalité du sens par rapport au préfixe : démultiplier, se décarrer. Le seul cas clair de compositionnalité transparente au départ semble être le verbe dédoubler qui aurait signifié au départ « ramener à l'unité (ce qui est double) », c'est-à-dire le contraire de doubler.

En résumé, sur ces 22 verbes, 19 d'entre eux sont sémantiquement opaques du point de vue du préfixe dès leurs premières attestations, la très grande majorité d'entre eux étant synonymes du simplex. L'émergence de cette opacité ne peut donc pas être expliquée par un processus diachronique. L'explication doit être trouvée en synchronie.

\subsection{LES DONNÉES SYNCHRONIQUES ACTUELLES NE PERMETTENT PAS D'ÉCARTER CES VERBES}

Deux explications synchroniques de l'existence de $\mathrm{V}_{\mathrm{DÉ}}$ comme découper, délaisser, ont été proposées, mais elles conduisent toutes les deux à considérer que ces verbes ne sont pas sémantiquement des mots construits.

La principale explication repose sur la notion d'intégration paradigmatique, développée initialement par Danielle Corbin (cf. Corbin, 1989b) ${ }^{4}$. Selon Gerhard-Krait (2000), le segment dé- aurait pu être ajouté à laisser simplement par un processus analogique formel pour intégrer ce verbe dans un paradigme de formes verbales elles-mêmes préfixées en DÉ. Cela revient à considérer que la forme préfixale serait sémantiquement vide dans délaisser, au moment de la formation du mot.

L'auteur envisage cependant une autre explication, voisine de l'intégration paradigmatique, à savoir la rénovation de forme, c'est-à-dire l'adjonction d'une forme préfixale pour servir à « renouveler des formes vieillies » (p. 125). Là encore, cet ajout est par hypothèse purement formel, la forme préfixale ayant une « non valeur [sémantique] ». L'auteur applique aussi cette hypothèse au verbe délaisser (p. 151).

\footnotetext{
${ }^{4}$ Pour une explicitation de cette notion, voir Apothéloz (2002 : 64-65).
} 
Mais, Gerhard-Krait note que le verbe laisser est polysémique ; de même, le verbe délaisser est lui-même polysémique ; et l'auteur indique brièvement que ces deux polysémies ne semblent pas coïncider. Pourtant, si l'on ajoute à la base laisser un segment morpho-phonologique qui n'est porteur d'aucune instruction sémantique, alors le résultat de cet ajout ne devrait rien changer par rapport aux propriétés sémantiques de laisser (un peu comme l'ajout de l'élément neutre dans l'addition : $0+5=5$ ). En conséquence, la polysémie de délaisser devrait être identique à celle de laisser.

Observant que ce n'est pas le cas, l'auteur fait alors l'hypothèse suivante : (p. 151, n65) : «Si toutefois tous les sens de laisser ne sont pas véhiculés par délaisser, il est possible également d'attribuer la différence à des spécificités dénominatives ». L'auteur entend par là que s'il y a des différences sémantiques éventuelles entre laisser et délaisser, alors elles sont arbitraires (les évolutions imprévisibles et contingentes du sens des mots), c'est-à-dire non imputables à la présence de la forme préfixale, puisque supposée sémantiquement vide. En d'autres termes, la différence sémantique entre délaisser et laisser serait sémantiquement contingente et ne découlerait pas de la présence de DÉ.

Or, comme nous allons le voir ci-après, le fait essentiel est là : l'ensemble des $\mathrm{V}_{\text {DÉ }}$ synonymes de leur simplex et ces simplex eux-mêmes sont les uns et les autres généralement polysémiques, et leurs polysémies ne coïncident pas. Si l'hypothèse de Gerhard-Krait est exacte, alors les différences sémantiques entre délaisser et laisser, découper et couper sont quelconques, sans lien avec la signification du préfixe DÉ. Si au contraire, les différences sémantiques observées ont un rapport avec le sens du préfixe DÉ, alors il ne sera plus possible de conclure que cette forme préfixale est vide de sens dans délaisser ou découper. La comparaison de la polysémie de ces $\mathrm{V}_{\mathrm{DÉ}}$ avec leur simplex s'avère donc le test crucial. Mais, pour mener à bien ces observations, nous avons tout d'abord besoin d'une hypothèse sémantique générale sur le préfixe DÉ.

En effet, nous avons vu ci-avant que ce préfixe était lui-même polysémique. Mais, Gerhard-Krait a montré que cette polysémie pouvait être subsumée par une instruction sémantique générale invariante que nous allons voir maintenant.

\subsubsection{Hypothèse sur l'instruction sémantique du préfixe DÉ-, inspirée de celle de F. Gerhard-Krait (2000)}

Nous n'avons évoqué jusqu'à présent que les cas où la base du $\mathrm{V}_{D E ́}$ est un verbe. Mais, le préfixe DÉ admet d'autres rapports catégoriels, notamment des verbes construits sur une base nominale comme désosser ou déterrer, ou bien des verbes construits sur une base adjectivale, comme dénasaliser (une voyelle) . $^{5}$

\footnotetext{
${ }^{5}$ Ce préfixe se rencontre aussi dans quelques noms construits sur des noms (déplaisir,
} 
L'hypothèse générale de Gerhard-Krait (2000), maintenue dans Gerhard-Krait (2012), vaut pour ces différents rapports catégoriels, auxquels correspondent différentes acceptions du préfixe. L'auteur postule que le préfixe DÉ évoque dans tous ses emplois une situation, notée «b $\mathrm{b}$, par rapport à laquelle une entité notée « $\mathrm{a}$ » (de nature quelconque) s'éloigne. L'invariant sémantique du préfixe DÉ a donc la forme suivante : «éloigner a de $\mathrm{b}$ ».

Cependant, cette notion d' « éloignement » ne convient que partiellement pour décrire un certain nombre d'emplois. Par exemple, dans un emploi comme leur voilier a démâté, il ne s'agit pas tant de dire que le mât a été "éloigné » du bateau, que de dire qu'il n'est plus à sa place, là où il était fonctionnel, c'est-à-dire qu'il est séparé du tout qu'il formait avec le bateau. De même, dans désaler l'eau de mer, il ne s'agit pas tant d'éloigner le sel de l'eau de mer; ce n'est pas un problème de distance entre le sel et l'eau. Il s'agit de séparer le sel de l'eau avec laquelle il formait un tout. En conséquence, nous décrirons plutôt l'invariant sémantique du préfixe DÉ en termes de relation de séparation, celleci pouvant localement s'interpréter comme un éloignement, un écart, une sortie d'un espace, etc. Nous faisons donc l'hypothèse que le préfixe DÉ signifie :

\section{« séparation d'une entité a d'une situation $b$ »}

où, je le rappelle, «b $»$ représente la situation de référence, et «a l'entité séparée de $« b$ ». Cet invariant sémantique n'est probablement pas assez précis, mais il sera suffisant pour les besoins de nos observations et de notre démonstration ciaprès. En tout cas, à la suite de l'auteur, nous insistons sur le fait que le procès exprimé par un $\mathrm{V}_{D E ́}$ est toujours à polarité initiale : on s'éloigne d'une situation de départ.

Cette situation par rapport à laquelle s'opère une séparation peut être définie à partir de la base verbale, comme dans désobéir. Le verbe obéir définit une situation, et le préfixe DÉ nous dit que l'on se sépare de cette situation d'où l'interprétation «ne pas obéir».

Mais, selon les $\mathrm{V}_{\mathrm{DÉ}}$ et selon leurs emplois, Gerhard-Krait souligne que les éléments « $\mathrm{a}$ » et « $\mathrm{b}$ » de l'instruction sémantique du préfixe sont représentés par des éléments très variables de la relation prédicative où se trouve le $V_{D E ́}$. Ils peuvent ainsi correspondre à des éléments de la structure argumentale du verbe, en l'occurrence son sujet et / ou son complément ${ }^{6}$. Ainsi, dans le train

déshonneur, etc.) et quelques adjectifs construits sur des adjectifs (défavorable, déloyal, etc.). Ceci dit, les propriétés sémantiques du préfixe dans ces emplois sont également subsumés par l'invariant sémantique proposé par Gerhard-Krait.

${ }^{6}$ Ce type d'hypothèse sur la variation de la portée de l'instruction sémantique d'un préfixe verbal est relativement partagée à l'heure actuelle. Je renvoie à Paillard (2004) et Némo (2010) pour des discussions générales. 
a déraillé, la situation initiale est définie par « le train est sur les rails », et le préfixe DÉ nous dit que l'entité «a » (ici le train) se sépare de cette situation, d'où l'interprétation que le train n'est plus sur les rails. Au contraire, dans Paul a désossé le gigot, la situation initiale est définie par la relation de localisation entre le référent de la base nominale du verbe et le référent du complément du verbe (l'os est dans le gigot); le préfixe DÉ nous dit que l'entité «a » (ici l'os) est séparé de cette situation de départ, d'où l'interprétation que l'on enlève l'os du gigot.

À travers ce bref parcours, nous voulons insister sur le fait que l'instruction sémantique du préfixe ne porte pas seulement sur la base du verbe, mais peut porter également sur les éléments de la structure argumentale du verbe. Nous avons là un premier niveau de variation des emplois du préfixe $\mathrm{e}^{7}$. Nous allons voir que l'explication des emplois opaques impose d'envisager un second niveau de variation de la portée sémantique du préfixe, à distinguer de celui évoqué ici.

Mais pour l'instant, revenons à notre problème central, l'existence supposée de relations de (quasi-) synonymie entre certains $\mathrm{V}_{\text {DÉ }}$ et leurs simplex (découper / couper, délaisser / laisser, etc.).

\subsubsection{Observation de la polysémie de quelques verbes en DÉ sémantiquement opaques}

Reprenons l'hypothèse de Gerhard-Krait concernant le verbe délaisser : au départ, l'adjonction de dé- à laisser serait un processus purement formel (intégration paradigmatique), puis par les hasards des évolutions de sens des mots, délaisser et laisser se seraient peu à peu différenciés dans leur polysémie. Notons d'emblée qu'en fait, dès le départ, la polysémie de délaisser est différente de celle de laisser ; ainsi, au moment des premières attestations de délaisser le simplex avait aussi l'acception « ne pas empêcher le déroulement de tel ou tel événement », sens que ne semble pas avoir eu le verbe délaisser. Mais, regardons les faits à l'heure actuelle.

S'il est exact que le verbe délaisser n'a aucun rapport en synchronie avec le sens actuel du préfixe DÉ, étant donné l'opacité sémantique de la forme préfixale, alors la diversité des emplois de délaisser, par différence avec celle

7 Notons que le préfixe DÉ- permet de construire aussi bien des verbes intransitifs (dérailler, décroitre) que des verbes transitifs directs (dénouer, désosser) ou indirects (désobéir, déplaire). De plus, lorsque la base est de nature verbale, celle-ci peut elle-même être intransitive (croître), transitive directe (nouer) ou indirecte (obéir). Ce préfixe semble donc relativement sous-déterminé par rapport aux propriétés syntaxiques des verbes qu'il permet de construire. C'est pourquoi dans la suite de cet article nous ne précisons pas les propriétés syntaxiques des verbes en discussion. 
de laisser, doit être sémantiquement quelconque par rapport au sens du préfixe ; idem pour découper, dépasser, etc. Voici ci-dessous un tableau représentatif de la diversité des emplois de délaisser et laisser:

LAISSER

laisse-le tranquille! on lui a laissé une

laissez-moi passer! part de gâteau / un allez, je vous laisse, mot

à bientôt !

ça a laissé des marques

ça laisse à désirer

laisser les volets fermés

laisser tomber ne me laisse pas ! le typhon a laissé derrière lui un paysage désolé Paul a laissé à ses enfants une fortune considérable

DÉLAISSER

I) a. Alain a laissé le piano pour la danse

b. Alain a délaissé le piano pour la danse
II) Paul délaisse son amie

III) C'est une profession qui est délaissée par les jeunes

IV) Paul délaisse son héritage ${ }^{8}$

Il apparaît qu'à l'heure actuelle le différentiel de polysémie entre les deux verbes est net. Cette différence est-elle pour autant contingente du point de vue du sens du préfixe DÉ actuel ? Examinons brièvement les différents emplois de délaisser. L'emploi I) où les deux verbes sont sémantiquement proches, exprime l'idée que Alain ne joue plus de piano, ce qui sous-entend qu'avant il jouait du piano. Donc, dans cet emploi, délaisser exprime une relation d'éloignementséparation entre Alain et l'activité de piano. L'emploi II) signifie qu'au départ il y avait une relation (affective) entre Paul et son amie ; ensuite, Paul s'est éloigné de son amie et le lien s'est distendu. Cet emploi de délaisser exprime donc aussi un éloignement-séparation entre Paul et son amie ; de même, l'emploi III) exprime une relation d'éloignement-séparation entre les jeunes et la profession en question. Enfin l'emploi IV) signifie que Paul décide de se séparer de la situation constituée par l'héritage auquel il avait droit.

Ainsi, l'ensemble des emplois de délaisser exprime une relation d'éloignement-séparation, par différence avec laisser. En effet, ce dernier admet par ailleurs des emplois sans rapport direct avec une idée d'éloignementséparation, voire l'inverse, comme dans laisser les volets fermés, proche de maintenir les volets fermés. Il apparait donc que la polysémie du verbe délaisser est entièrement circonscrite par la signification du préfixe DÉ actuel. Chacun des emplois de délaisser met en jeu une relation où une entité «a » (Alain / les jeunes / Paul) est séparée ou se sépare d'une situation d'origine « $\mathrm{b}$ » dont elle faisait partie, ce qui correspond exactement à l'invariant sémantique du préfixe DÉ mis au jour ci-avant. L'hypothèse de Gerhard-Krait sur délaisser

\footnotetext{
${ }^{8}$ Emploi juridique signifiant « renoncer à la possession de qqch ».
} 
est donc contredite par les faits : le différentiel entre la polysémie de laisser et celle de délaisser n'est en aucune façon arbitraire, contingent par rapport au sens du préfixe DÉ actuel. Par conséquent, l'explication même de la création du mot délaisser en termes d'intégration paradigmatique est contredite. Le préfixe DÉ est manifestement présent autant sémantiquement que formellement dans délaisser.

Intéressons-nous maintenant au verbe découper. Comme pour délaisser, nous observons que la polysémie de ce $\mathrm{V}_{\mathrm{DÉ}}$ est différente de celle de son simplex. Certes, ils partagent quelques acceptions synonymes, mais ils ont aussi des emplois sémantiquement éloignés.

COUPER

DÉCOUPER

\begin{tabular}{|c|c|c|c|}
\hline $\begin{array}{l}\text { couper l'herbe / du } \\
\text { bois / les cheveux }\end{array}$ & $\begin{array}{l}\text { couper un morceau } \\
\text { de pain }\end{array}$ & & \\
\hline $\begin{array}{l}\text { Thomas s'est } \\
\text { coupé avec la lame } \\
\text { couper les crédits } \\
\text { couper la parole / } \\
\text { la tête } \\
\text { ça coupe l'appétit } \\
\text { couper à travers } \\
\text { champs } \\
\text { les gendarmes ont } \\
\text { coupé la nationale } \\
\text { à la hauteur de } \\
\text { Montargis }\end{array}$ & $\begin{array}{l}\text { il a coupé la corde } \\
\text { couper un passage } \\
\text { (d'un texte, d'un } \\
\text { interrogatoire) } \\
\text { ils ont coupé le } \\
\text { courant } \\
\text { couper le salon en } \\
\text { deux } \\
\text { tu ne vas pas y } \\
\text { couper } \\
\text { se couper de sa } \\
\text { famille }\end{array}$ & $\begin{array}{l}\text { I) a. couper le } \\
\text { poulet } \\
\text { b. découper le } \\
\text { poulet } \\
\text { II) a. couper } \\
\text { un article de } \\
\text { journal } \\
\text { b. découper } \\
\text { un article de } \\
\text { journal }\end{array}$ & 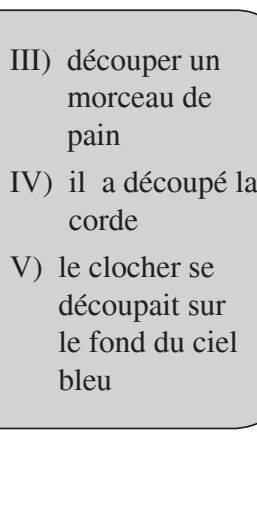 \\
\hline
\end{tabular}

Encore une fois, demandons-nous si cette différence dans la polysémie des deux verbes est contingente du point de vue du sens du préfixe DÉ actuel, ou pas ? Examinons brièvement les différents emplois de découper. Dans l'acception I), nous avons une situation de départ constituée par le poulet en une seule pièce, et à l'issue du procès, il est en plusieurs morceaux, ce qui revient à dire que l'on a séparé les différentes parties du poulet. De même, dans l'acception II), à l'issu du procès, on a séparé l'article du reste du journal. Idem pour les acceptions III) et IV). L'interprétation de l'acception V) est à première vue moins évidente, car il n'y a pas de séparation matérielle, comme dans découper le poulet. Cependant, sur un mode métaphorique, on retrouve le même sens : cet emploi renvoie à l'idée d'un motif qui se détache, se distingue par rapport à un fond, ce qui revient à dire qu'il se sépare nettement de ce fond ; cette séparation ne correspond certes pas à un éloignement dans l'espace, mais 
il y a bien l'expression d'une frontière nette entre le motif et le fond, c'est-àdire une séparation d'une entité par rapport à un tout dont elle fait partie. A nouveau, comme pour délaisser, nous voyons que l'ensemble des emplois du verbe découper correspondent à l'instruction sémantique du préfixe DÉ actuel.

Nous terminerons ces brèves observations par le verbe détourner. Ce verbe est intéressant car il présente un degré d'opacité sémantique dans la compositionnalité du sens plus important que délaisser ou découper. En effet, cette opacité porte également en partie sur le sens de la base : l'existence d'un rapport sémantique entre détourner dans détourner une somme d'argent et le moteur tourne ne va pas de soi. Voyons néanmoins ce qu'il en est des différentes acceptions de ce $\mathrm{V}_{\text {DÉ }}$ par rapport au sens du préfixe DÉ.

TOURNER

DÉTOURNER

\begin{tabular}{|c|c|c|c|}
\hline & & & $\begin{array}{l}\text { II) détourner un } \\
\text { cours d'eau }\end{array}$ \\
\hline $\begin{array}{l}\text { la terre tourne } \\
\text { ma tête tourne } \\
\text { le moteur tourne } \\
\text { tourner le bois } \\
\text { tourner à gauche } \\
\text { tourner la } \\
\text { manivelle } \\
\text { tourner autour de } \\
\text { qqch } \\
\text { tourner un film }\end{array}$ & $\begin{array}{l}\text { tourner qqch en } \\
\text { dérision } \\
\text { ça lui a tourné la } \\
\text { tête } \\
\text { tourner la page } \\
\text { tourner son } \\
\text { fauteuil vers la } \\
\text { fenêtre } \\
\text { tourner toutes ses } \\
\text { pensées vers qqn } \\
\text { tourner court } \\
\text { se tourner vers } \\
\text { qqch /qqn }\end{array}$ & $\begin{array}{l}\text { I) a. Il tourna } \\
\text { le regard pour } \\
\text { éviter de croiser } \\
\text { celui de Marie } \\
\text { b. Il détourna } \\
\text { le regard pour } \\
\text { éviter de croiser } \\
\text { celui de Marie }\end{array}$ & $\begin{array}{l}\text { III) détourner un } \\
\text { avion } \\
\text { IV) détourner la } \\
\text { conversation / } \\
\text { l'attention / les } \\
\text { soupçons } \\
\text { V) détourner le } \\
\text { sens d'un texte } \\
\text { VI) détourner qqn } \\
\text { de sa route / de } \\
\text { ses occupations } \\
\text { VII) détourner une } \\
\text { grosse somme } \\
\text { d'argent }\end{array}$ \\
\hline & & & $\begin{array}{l}\text { VIII) se détourner } \\
\text { de qqch / qqn }\end{array}$ \\
\hline
\end{tabular}

L'emploi I), le seul sens que nous ayons recensé, illustrant une synonymie avec le simplex correspond à une situation où le regard est écarté, séparé de son destinataire initial. L'emploi II) détourner un avion signifie éloigner l'avion de sa trajectoire initiale ; de même, détourner un cours d'eau, c'est le sortir de sa situation initiale, c'est-à-dire de son lit naturel ; détourner une conversation, c'est imposer un sujet de conversation qui sort la conversation de son sujet initial ; détourner le sens d'un texte, c'est donner au texte un sens qui s'éloigne $\mathrm{du}$ sens original ; détourner quelqu'un de ses occupations, c'est amener cette personne à ne plus avoir ces occupations, c'est-à-dire s'en éloigner ; détourner une somme d'argent, c'est faire en sorte que l'argent n'aille pas à son 
destinataire initial, donc écarter cette somme d'argent de cette situation initiale ; se détourner de qqn, c'est rompre la relation préexistante avec cette personne, et donc se séparer de cette situation initiale.

Encore une fois, nous constatons que les différentes acceptions de ce $V_{D E ́}$ correspondent à chaque fois à l'invariant sémantique du préfixe DÉ. Le verbe tourner quant à lui ne met pas particulièrement en jeu une idée d'éloignementséparation dans ses différents emplois comme dans tourner un bouton / un film. Et nous constatons que l'emploi de détourner dans ces derniers emplois ne marche pas, sauf justement à modifier l'interprétation pour retrouver le sens du préfixe DÉ : détourner un film signifierait nécessairement quelque chose comme « lui donner une signification / un objectif éloigné de celui qu'avait donné son auteur »; c'est précisément ce que l'on observe dans les données attestées, comme dans l'exemple suivant :

(7) détourner un film-référence permet de faire passer des messages-clefs sérieux avec un ton léger, un décalage humoristique.

À l'issu de ce bref parcours, nous nous retrouvons dans une situation a priori paradoxale : d'un côté, le sens de dé-dans délaisser ou découper est intuitivement opaque pour les locuteurs, ce qui laisserait penser que ces verbes ne contiennent pas le préfixe DÉ en synchronie ; d'un autre côté, nous venons de voir que la polysémie de ces $\mathrm{V}_{D E ́}$ correspond exactement à l'invariant sémantique du préfixe DÉ actuel, ce qui amène à conclure qu'ils contiennent le préfixe DÉ. Une analyse sémantique synchronique satisfaisante doit pouvoir rendre compte de ces deux aspects à la fois. C'est ce que nous nous proposons de présenter maintenant.

\section{PROPOSITION D'ANALYSE SYNCHRONIQUE DES EMPLOIS OPAQUES DU PRÉFIXE DÉ}

Rappelons que nous étudions ici des $\mathrm{V}_{\mathrm{DÉ}}$ dont la base est elle-même de nature verbale. A ce titre, la base du verbe préfixé est de nature prédicative ; en conséquence, elle exprime une relation (un prédicat est fondamentalement un relateur). Par conséquent, le préfixe DÉ porte sur un élément exprimant une relation. À partir de là, notre analyse reposera sur deux hypothèses complémentaires de cette première hypothèse :

a) Les invariants sémantiques du préfixe et de la base sont des formes ; plus précisément, nous adoptons l'hypothèse des formes schématiques développée dans le cadre de la théorie des opérations prédicatives et énonciatives, initiée par Antoine Culioli (cf. Culioli (1990-1999). Ces formes schématiques correspondent à des invariants sémantiques ${ }^{9}$, en

\footnotetext{
${ }^{9}$ L'hypothèse des invariants sémantiques n'est pas spécifique à ce cadre théorique (cf.
} 
deçà de la variation sémantique liée à la diversité des emplois de l'unité considérée ${ }^{10}$;

b) le préfixe verbal DÉ est lui-même de nature prédicative ${ }^{11}$. En tant qu'élément prédicatif, le préfixe est conçu lui aussi comme exprimant une relation. Dès lors, analyser la relation sémantique entre DÉ et la base verbale revient à analyser une relation entre des relations. En conséquence, un $\mathrm{V}_{\mathrm{DE}}$ correspond par hypothèse à la combinaison de deux éléments prédicatifs, c'est-à-dire un prédicat complexe.

Les types de relations exprimées par les formes schématiques des bases verbales, et le nombre d'éléments mis en jeu dans ces formes schématiques est éminemment variable. Cependant, dans le cadre de la combinaison avec un préfixe verbal, on peut, en simplifiant, ramener la forme de ces relations à un schéma général mettant en jeu deux éléments ${ }^{12}$. Nous pouvons représenter schématiquement chacune de ces deux relations comme suit :
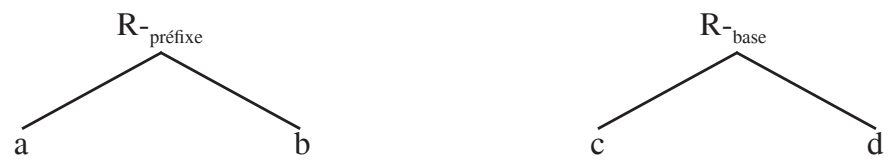

Dans le cas du préfixe DÉ-, nous rappelons que la lettre « $\mathrm{b} »$ représente ce par rapport à quoi il y a éloignement-séparation; la lettre « $\mathrm{a}$ » représente ce qui est dans une relation d'éloignement-séparation par rapport à b. Pour le préfixe comme pour la base verbale, les éléments mis en relations au niveau des formes schématiques peuvent s'incarner dans des éléments très variables de la relation prédicative (les différents arguments du verbe). Nous l'avions déjà vu avec le préfixe sur des exemples comme dérailler ou désosser. Nous ne développerons pas ce point pour la base verbale, car cela n'est pas centrale dans notre analyse.

Le principe explicatif synchronique de la différence entre un $V_{D E ́}$ perçu comme sémantiquement transparent (dénouer) et un $\mathrm{V}_{\mathrm{DÉ}}$ perçu comme opaque du point de vue du préfixe (découper) repose sur l'idée qu'il existe non pas un

plus largement les travaux en sémantique instructionnelle). Ce qui importe ici est de pouvoir concevoir ces invariants sémantiques comme des formes.

${ }^{10}$ En conséquence, la base sur laquelle porte l'instruction sémantique du préfixe est potentiellement polysémique ; sans cette hypothèse, on ne peut pas rendre compte de la diversité des emplois d'un verbe comme découper ou détourner.

${ }^{11}$ Une telle hypothèse n'a rien de nouveau dans le domaine des préfixes verbaux. On la trouve par exemple mise en œuvre dans Rousseau (1995). Elle est également adoptée depuis longtemps dans l'étude des préverbes du russe (cf. D. Paillard, 2010). On la trouve également dans Lieber et Baayen (1993) pour les préfixes verbaux du néerlandais.

12 Pour un développement argumenté de ce point, cf. Jalenques (2000, chapitre 8) ; pour une analyse similaire concernant les préverbes du russe, voir également Paillard (2010). 
mais au moins deux types de relations possibles entre la forme schématique du préfixe verbal et celle de la base verbale. Nous allons maintenant examiner chacun d'eux.

\subsection{PREMIER CAS : COMPOSITIONNALITÉ ADDITIONNELLE (CORRESPOND AUX EMPLOIS TRANSPARENTS)}

Considérons l'exemple suivant :

(8) Paul a dénoué sa cravate

Ici, nous avons affaire à une compositionnalité du sens transparente entre le préfixe et la base verbale. Elle correspond à une compositionnalité « additionnelle » des formes schématiques. Nous entendons par là que l'instruction sémantique du préfixe s'ajoute de l'extérieur à la relation exprimée par la base verbale (intégrant ses éventuels arguments); en d'autres termes, elle s'applique à la relation exprimée par la base considérée globalement, comme un bloc indécomposable. Ceci a pour conséquence, et cela est le point crucial, qu'ici nous avons deux procès exprimés correspondant à chacun des deux prédicats (le préfixe et la base verbale) :

- le procès correspondant à la relation exprimée par c-R- ${ }_{\text {base }}-\mathrm{d}$ (Paul a noué sa cravate); cette relation correspond globalement à l'élément « $\mathbf{b}$ » de la relation exprimée par DÉ-. Cette relation, correspondant ici à un procès télique, débouche sur la situation « la cravate est nouée »;

- le procès correspondant à la relation exprimée par le préfixe DÉ : on s'éloigne de la situation « $\mathrm{b} »$, donc on se sépare de la situation « la cravate est nouée ».

Cette relation concaténatoire entre les deux formes schématiques peut être représentée comme suit :

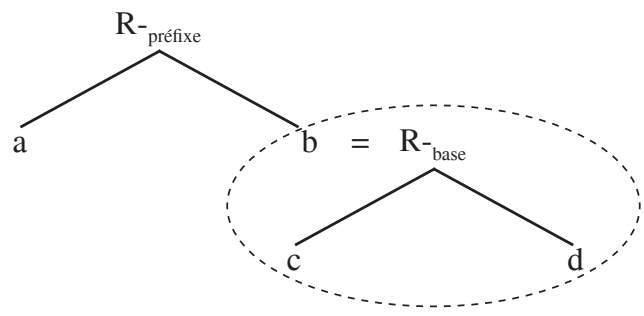

La glose est donc la suivante : on s'éloigne-sépare de la situation (résultante) définie par nouer la cravate, d'où l'interprétation qu'à l'issue du second procès (exprimé par DÉ), la cravate n'est plus nouée. 


\subsection{DEUXIÈME CAS : COMPOSITIONNALITÉ PAR RECOUVREMENT DES FORMES SCHÉMATIQUES (CORRESPOND AUX EMPLOIS OPAQUES)}

Considérons maintenant l'exemple suivant :

(9) Paul a découpé le losange en suivant les pointillés

Le point clé ici, nous l'avons vu à la section précédente, est que l'événement lui-même exprime une relation d'éloignement-séparation. L'explication est la suivante : la relation exprimée par la base verbale est in-formée par l'instruction sémantique du préfixe. Nous voulons dire par là que la relation exprimée par couper prend la forme de la relation exprimée par DÉ, c'està-dire une relation d'éloignement-séparation. C'est le fait même de penser les instructions sémantiques comme des formes (schématiques) qui nous permet cette hypothèse. Précisons que «prendre la forme de » ne signifie en aucune façon que la forme schématique de couper se réduit à celle du préfixe DÉ ; cela signifie que la forme schématique de couper est déformée de telle sorte qu'elle manifeste la forme de la forme schématique du préfixe.

Par différence avec la compositionnalité transparente du sens, cette fois-ci, la forme schématique du préfixe ne s'applique plus globalement, de l'extérieur, à la relation fondée à partir de la base verbale; elle s'applique directement à la forme même de la relation exprimée par la base verbale, ce que l'on peut représenter comme suit :

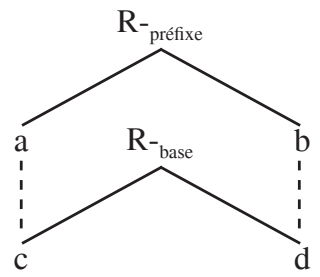

En conséquence, le procès exprimé par la base verbale (c-R-d) prend la forme d'un procès exprimant en lui-même un éloignement-séparation ( $a-R-b)$ - c'est bien la signification du procès que l'on observe en (9). C'est pourquoi, ici, il n'y a pas deux procès en jeu (comme dans dénouer la cravate). Il y a un seul procès qui possède à la fois les caractéristiques de la relation exprimée par couper et les caractéristiques de la relation exprimée par DÉ-. Ici, le verbe ne signifie pas se séparer de la situation exprimée par couper; il signifie couper de telle sorte que l'on sépare quelque chose de quelque chose. En l'occurrence, découper le losange en suivant les pointillés signifie bien que l'on sépare la forme du losange de la feuille de papier sur laquelle il était dessiné.

C'est parce qu'il y a construction d'un seul procès à partir de la combinaison de deux instructions sémantiques qu'intuitivement la 
compositionnalité du sens est opaque. Cette opacité ne résulte donc en aucune façon d'une perte des propriétés sémantiques du préfixe, mais résulte d'une combinaison intriquée entre les relations exprimées par le préfixe DÉ- et la base couper. Prenons encore l'exemple suivant :

(10) Paul délaisse son amie

À nouveau, par différence avec dénouer la cravate, il n'y a pas deux procès combinés, dont le premier serait exprimé à partir de laisser et le second à partir du préfixe DÉ. Il y a un seul procès, exprimé globalement par délaisser. L'événement exprimé correspond à une relation d'éloignement-séparation parce qu'encore une fois la relation exprimée par la base verbale est in-formée par l'instruction sémantique du préfixe. Le $\mathrm{V}_{\mathrm{DÉ}}$ signifie laisser de telle sorte que l'on se sépare d'une situation initiale (la relation affective entre Paul et son amie). Parce que la forme schématique du préfixe informe la forme schématique de la base verbale, le préfixe constitue l'élément principal du prédicat complexe.

Cette analyse permet en outre de rendre compte du fait que la relation de synonymie (locale) entre le $\mathrm{V}_{\mathrm{DÉ}}$ et le simplex se rencontre majoritairement avec des verbes exprimant eux-mêmes une idée d'éloignement-séparation. Dans la mesure où la compositionnalité par recouvrement des formes schématiques implique que la relation exprimée par la base prenne la forme de la relation exprimée par le préfixe, encore faut-il que cette relation exprimée par la base soit in-formable par celle du préfixe. Lorsqu'un verbe exprime dans son sémantisme une idée d'éloignement-séparation, il est déjà prédisposé à prendre la forme de la relation exprimée par DÉ. Ainsi, des verbes comme couper ou laisser mettant en jeu une idée de séparation dans une partie de leurs emplois, leur combinaison avec DÉ n'entrainera pas une grande différence de sens avec le simplex lorsqu'ils seront in-formés par la relation du préfixe $\mathrm{DE}^{13}$. En conséquence, dans ces verbes, la forme préfixale dé- n'est en aucune façon vide

${ }^{13}$ Ces cas de quasi-synonymie entre le préfixe et la base verbale elle-même pourraient a priori faire penser au phénomène de multi-exponence : Cette notion renvoie à la situation où plusieurs marqueurs, généralement flexionnels, sont présents dans un même mot alors qu'ils sont porteurs d'information(s) grammaticale(s) identiques, d'où un effet de redondance (cf. Caballero \& Harris, A. C. (2012). Or, les cas de synonymie étudiés ici ne sont redondants qu'en apparence, puisque la polysémie du verbe en DÉ est toujours différente de celle de son simplex. De plus, contrairement à la multi-exponence, cette proximité sémantique concerne des objets linguistiques de nature et de niveau différents : un objet affixal (un préfixe) combiné à une unité lexicale (la base). Enfin, il n'y a jamais redondance entre le préfixe et la base dans la mesure où le sens du $V_{D E}$ n'est jamais identique à celui du simplex. Il n'y a pas redondance de l'information mais « résonance » partielle entre deux instructions sémantiques, conduisant à l'émergence de valeurs sémantiques singulières. Les phénomènes étudiés ici ne peuvent donc être ramenés à des cas de multi-exponence. 
de sens (hypothèse de l'intégration paradigmatique de Gerhard-Krait), elle est au contraire pleinement porteuse du sens du préfixe DÉ.

Enfin, cette analyse permet d'expliquer simplement le cas de $V_{D E ́}$ dont nous n'avons pas encore parlé jusqu'à maintenant, à savoir des $V_{\text {DÉ }}$ admettant à la fois des emplois compositionnellement transparents et opaques par rapport au préfixe. Prenons l'exemple du verbe dédoubler, dans les deux emplois attestés suivants :

(11) a. Pendant qu'il [le moteur] chauffe, je déferle la grand-voile, vérifie les écoutes de génois, je dédouble les amarres.

b. À Paris-Diderot, nous avons dédoublé les TD pour réduire les effectifs par cours.

En (11a), l'emploi du $V_{D E ́}$ exprime l'idée que l'on avait au départ doublé les amarres (deux cordages passés en double) et que maintenant, on passe de deux cordages à un cordage, c'est-à-dire l'inverse de doubler les amarres. En (11b), le $\mathrm{V}_{\mathrm{DE}}$ signifie au contraire qu'étant donné une situation de départ où l'on a un nombre $\mathrm{n}$ de $\mathrm{TD}$, à l'issue du procès on a deux fois plus de TD. Ici, le $\mathrm{V}_{\mathrm{DE}}$ ne signifie pas « le contraire de doubler », il est sémantiquement proche de doubler, d'où l'opacité intuitive du sens de DÉ. Ceci amène certains dictionnaires (le TLF) à considérer ces deux emplois comme relevant de deux verbes homonymes.

Notre analyse permet de montrer qu'il s'agit d'un seul et même $V_{D E ́}$, mais illustrant selon ses emplois les deux relations entre la forme schématique du préfixe et celle de la base verbale que nous avons présentées. En (11a), nous avons affaire à une compositionnalité additionnelle des deux formes schématiques, d'où l'interprétation " s'éloigner de la situation résultante exprimée par doubler $»$. Au contraire, en (11b), nous avons affaire à une compositionnalité par superposition des deux formes schématiques, l'instruction sémantique du verbe doubler étant in-formée par celle du préfixe, d'où l'interprétation « doubler de telle sorte que cela mette en jeu une séparation ». Et, précisément, dédoubler les $T D$, cela signifie que l'on sépare en deux les groupes initiaux d'étudiants constituant ces TD et non simplement que l'on ajoute des TD aux TD de départ. Cette différence devient perceptible dans la comparaison entre doubler la garde et dédoubler la garde. Dans ce dernier cas, le $\mathrm{V}_{\mathrm{DÉ}}$ ne signifie pas que l'on met deux fois plus de gardes pour surveiller le bâtiment, mais que l'on sépare le groupe de départ des gardes en deux sous-groupes.

Le verbe démaigrir, restreint essentiellement à des emplois techniques, présente la même variation. Il peut signifier tantôt « diminuer la largeur d'un objet », tantôt « grossir », c'est-à-dire son contraire. Dans notre analyse, la valeur " grossir » correspond à la composition additionnelle des formes schématiques de $D E$ - et maigrir : on s'éloigne-sépare de la situation exprimée 
par maigrir, d'où la valeur « contraire de maigrir »; quant à la valeur « diminuer la largeur » elle correspond à la compositionnalité par superposition des formes schématiques de DÉ- et maigrir, d'où la glose « faire maigrir de telle sorte que l'on sépare quelque chose du tout », comme dans démaigrir une poutre qui signifie « enlever de la matière afin de rendre la poutre moins épaisse ». Il n'y a donc rien d'homonymique entre ces deux emplois de démaigrir mais deux relations sémantiques différentes entre le préfixe et sa base.

À l'issue de ce bref parcours, qu'en est-il de l'ensemble des $22 \mathrm{~V}_{\text {DÉ }}$ synonymes de leur simplex que nous avons listés en 2.1. ? En l'état actuel de nos observations, l'analyse que nous proposons fonctionne bien pour 19 de ces 22 verbes. Pour trois d'entre eux, nos conclusions sont pour l'instant incertaines : dénommer, détremper et dépeindre. En effet, il ne va pas de soi que ces trois verbes mettent en jeu une séparation par rapport à une situation initiale. Ceci dit, observons qu'une séquence comme Paul a dépeint une fleur sur le mur n'a pas le même sens que Paul a peint une fleur sur le mur. Cette dernière séquence tend à signifier que Paul a créé le dessin d'une fleur sur le mur. Avec dépeindre, cette interprétation n'est pas possible : La séquence signifiera plutôt «Paul a décrit et représenté avec des mots ce qui était dessiné sur le mur ». On retrouve donc une distance, c'est-à-dire une séparation, entre ce qui est dépeint (une représentation « peinte » dans un discours) et l'objet lui-même (la peinture qui est sur le mur). Cette séparation est peut-être due à la présence du préfixe DÉ. Seule une étude sémantique plus approfondie des emplois de ce verbe ainsi que de dénommer et détremper permettra de trancher.

\section{BILAN}

À l'issu de cette brève exploration de verbes en DÉ intuitivement perçus comme non compositionnels du point de vue du préfixe (découper, délaisser, par différence avec dénouer, déplaire), il apparaît que ces verbes sont en fait pleinement compositionnels ; mais, ils illustrent un autre type de compositionnalité du sens, où la relation exprimée par la base verbale prend la forme de la relation exprimée par le préfixe DÉ.

Nous insistons sur le fait que les deux types de compositionnalité du sens que nous avons mis au jour pour le préfixe DÉ dans la dernière partie relèvent d'un niveau de combinatoire sémantique différent de celui envisagé en 2.3.1. où nous avions indiqué que l'instruction sémantique du préfixe peut porter sur la base verbale ou bien sur les arguments du verbe de façon variable.

Nous avons vu que la clé pour mettre au jour le second type de compositionnalité du sens illustré par découper ou délaisser a reposé sur la prise en compte de la polysémie de ces verbes en DÉ, en la comparant avec la polysémie de leurs simplex respectifs couper, laisser, qui s'est avérée différente à chaque fois. En raison même de cette différence, la relation de synonymie 
intuitivement perçue entre découper et couper est finalement illusoire, car locale à seulement quelques acceptions de ces deux verbes, en l'occurrence des acceptions qui correspondent au sens du préfixe DÉ.

La situation présentée par les verbes comme découper ou délaisser apparaît a posteriori paradoxale : le préfixe DÉ apparait sémantiquement opaque, et donc a priori absent, dans les verbes où il est au contraire le plus présent, puisqu'il en constitue le prédicat principal (découper signifie avant tout «séparer un ou des éléments d'un tout»). L'opacité du sens de dé- que l'on perçoit dans découper, par différence avec dénouer, correspond en fait à une «illusion d'optique », due au fait que l'on cherche le sens du préfixe là où il ne se trouve pas.

Si l'on cherche dans découper une idée d'éloignement ou séparation appliquée à couper, on ne la trouvera pas ; en effet, découper ne signifie pas «le contraire de couper». Mais, si l'on cherche cette instruction sémantique au niveau du sens global du verbe, alors cette idée de séparation apparaît immédiatement (découper le poulet, cela signifie bien «en séparer les différentes parties constitutives »). Une fois que l'on accepte de regarder le sens de découper dans sa globalité, alors il est intuitivement perceptible que ce verbe exprime une idée d'éloignement-séparation, c'est-à-dire le sens même du préfixe DÉ. Finalement, le sens du préfixe dé- dans découper n'est en aucune façon opaque. Simplement, il se manifeste à un niveau où on ne l'attend / cherche pas, parce que l'observation est biaisée par un a priori sur la compositionnalité du sens, qui n'existerait que sous forme d'une relation additionnelle-concaténatoire. Nous avons ainsi montré que des verbes comme découper, délaisser ne sont en aucune façon irréguliers, mais manifestent une compositionnalité du sens d'un type différent.

On nous rétorquera peut-être que ce mode de compositionnalité du sens ne semble plus guère productif avec le préfixe DÉ à l'heure actuelle. Certes, d'après nos observations, la création de mots ou d'emplois de mot illustrant ce fonctionnement semble marginale avec DÉ. Cependant, elle est non nulle, donc le mode de construction semble encore disponible ${ }^{14}$. Mais, de toute façon, il

${ }^{14}$ Sur le modèle du verbe dédoubler, on relève la série de verbes détripler, déquadrupler, déquintupler, désextupler de création relativement récente $\left(20^{\mathrm{e}}\right.$ siècle pour détripler et les deux derniers semble-t-il). Voici un exemple attesté :

- maintenant je pige pourquoi j'arrive à faire tant de trucs en même temps. Merci Gustave grâce à toi je sais que je ne suis pas hyperactive mais seulement que je me désextuple.

Nous avons aussi relevé quelques emplois attestés, illustrant le même type de compositionnalité du sens, pour les verbes dépurifier et désenlever:

- Le grand nettoyage de printemps passe aussi par une cure détox qui va dépurifier votre corps alourdi par les toxines accumulées pendant l'hiver ;

- ca reste en position plein phare, il faut reappuyer pour désenlever les pleins phares !!! 
n'en reste pas moins que ces verbes manifestent un fonctionnement sémantique parfaitement régulier, et surtout manifestent pleinement les propriétés sémantiques du préfixe DÉ actuel. A ce titre, ils peuvent difficilement être écartés de l'ensemble des verbes considérés comme préfixés en DÉ à l'heure actuelle.

De plus, cette hypothèse d'une variation dans les relations sémantiques possibles entre le préfixe et la base verbale se retrouve au moins pour un autre préfixe en français, le préfixe RE. Elle ne peut donc pas être considérée comme ad hoc. En effet, cette analyse de la compositionnalité du sens pour les emplois opaques du préfixe DÉ rejoint l'analyse qu'avait proposée Jalenques (2000a et 2001) concernant le préfixe RE. L'auteur avait montré qu'une part importante des emplois opaques du préfixe RE correspond à une situation comparable à celle vue ici, où l'instruction sémantique du préfixe RE vient in-former la relation exprimée par la base verbale.

Prenons tout d'abord un emploi sémantiquement transparent du préfixe RE. Dans Paul a refait ses lacets, dans un contexte où les lacets étaient défaits, le préfixe exprime, pour simplifier, l'idée de mettre fin à une situation transitoire, d'où l'idée de retour à un état initial ; cet état initial étant défini à partir de ce que signifie faire ses lacets. Ici, l'instruction sémantique de RE s'ajoute de façon concaténatoire à la relation exprimée par faire (les lacets). Par contre, dans l'emploi le cycliste a refait son retard, la relation sémantique entre le préfixe et la base verbale est toute autre : cette fois-ci, la relation exprimée par faire est informée par la relation sémantique exprimée par RE. Le verbe peut être glosé par « faire quelque chose de telle sorte que cela s'interprète comme mettant fin à une situation transitoire », cette situation transitoire étant exprimée par le complément du verbe (le cycliste a pris du retard sur ses concurrents). Cela correspond précisément ce que l'on observe : refaire son retard signifie «mettre fin au retard» (refaire ses lacets ne signifie pas «mettre fin à l'existence des lacets »). On retrouve donc le même type de variation de la compositionnalité du sens entre les instructions sémantiques du préfixe RE et de la base verbale, à savoir une composition par addition ou par superposition.

Cette analyse qui postule au moins deux types de compositionnalité du sens dans les verbes préfixés semble donc constituer une hypothèse de portée plus générale que celle concernant le seul préfixe DÉ. Il reste bien sûr à la tester sur d'autres préfixes verbaux en français et dans d'autres langues. Dans son principe général, cette hypothèse d'une variation des relations possibles entre la forme schématique d'un préfixe et de sa base verbale rejoint les analyses de D. Paillard sur les préverbes du russe (cf. Paillard, 2004 et 2010). 


\section{RÉFÉRENCES}

Amiot, D. (2008), « La catégorie de la base dans la préfixation en dé », in Fradin, B. (éd.), La raison morphologique. Hommage à la mémoire de Danielle Corbin, Amsterdam/Philadelphia : Benjamins, 1-15.

Apotheloz, D. (2007), « Procès centrifuge et procès centripète : RE- DÉS- et l'antonymie directionnelle en français », in Hathout N. \& Montermini F. (éds), Morphologie à Toulouse. Actes du colloque international de Morphologie "4èmes Décembrettes". München : Lincom Europa (LSTL), 149-169.

Apotheloz, D. (2002), La construction du lexique français, Paris : Ophrys.

Apotheloz, D. et Boyé, G. (2004), « Remarques sur la compositionnalité en morphologie », Verbum, 26/4, 375-385.

Auroux S. (1984), « D’Alembert et les synonymistes », Dix-huitième siècle, 16, 93-108

Caballero, G. \& Harris, A. C. (2012), “A Working Typology of Multiple Exponence : Cross-linguistic Variation and Theoretical Implications", in Kiefer, F., Ladanyi M., Siptar P. (éds.), Current Issues in Morphological Theory, Selected Papers from the $14^{\text {th }}$ International Morphology Meeting, Budapest, May 13-16, 2010, 163-188.

Corbin, D. (1989a), « La place de l'histoire dans une morphologie synchronique », Acta Universitatis Wratislaviensis $n^{\circ}$ 1064, Romanica Wratislaviensia XXX : 51-67. Wroclaw/Paris : Nizet.

Corbin, D. (1989b), « Contraintes et création lexicales en français », L'Information Grammaticale, 42, 35-43.

Culioli, A. (1990-1999), Pour une linguistique de l'énonciation. Tome 1 à 3, Paris : Ophrys.

Gerhard-Krait, F. (2000), La préfixation en dé(s)- : formes construites et interprétations, thèse de doctorat, Université Marc Bloch, Strasbourg II.

Gerhard-Krait, F. (2012), « Du nom place aux verbes déplacer et replacer : quelques questions de legs et d'appropriations sémantiques », CORELA, Numéros thématiques Langue, espace, cognition, URL : http://corela.edel.univ-poitiers.fr/ index.php?id=2790, publié en ligne le 18 décembre 2012.

Jalenques, P. (2000a), « Pour une approche non concaténatoire de la construction du sens des verbes préfixés : analyse de l'emploi dit « explétif « du préfixe re- en français contemporain », in Actes du XXIIe Congrès international de Linguistique et Philologie romanes (Bruxelles, 23-29 juillet 1998), 305-316.

Jalenques, P. (2000b), « Contribution à l'étude du préfixe RE en français contemporain : pour une analyse compositionnelle du verbe regarder », thèse de doctorat, Université Paris 7. 
Jalenques, P. (2001), « Quand la diachronie renvoie à la synchronie : étude des emplois idiomatiques du préfixe RE en français (reconnaître, remarquer, regarder, etc. ) », Recherches linguistiques de Vincennes, 30, 39-61.

Lieber, R. \& Baayen, H. (1993), "Verbal prefixes in Dutch : a study in lexical conceptual structure", Yearbook of morphology, 51-78.

Muller, Cl. (1990), « Contraintes de perception sur la productivité de la préfixation verbale en dé- négatif », Travaux de linguistique et de philologie, XXVIII, 172-191.

Némo, F. (2008) , «Pour une sémantique non combinatoire en morphologie (et syntaxe) : introduction aux notions de pool et d'intégration sémantique », Colloque Grammaire, morphologie, sémantique, même combat?, 25 septembre 2008.

Némo, F. (2010), « Routines interprétatives, constructions grammaticales et constructions discursives, Estudos Linguísticos/Linguistic Studies, 5, Edições Colibri/CLUNL, Lisboa, 35-53.

Paillard, D. (2004), « À propos des verbes préfixés », Slovo, 30-31, INALCO, 13-44.

Paillard, D. (2010), « La notion de prédicat complexe », Faits de langues. Les Cahiers, 2, 197-228.

Rousseau, A. (éd.) (1995), Les préverbes dans les langues d'Europe - Introduction à l'étude de la préverbation, Lille : Presses universitaires du Septentrion. 